\title{
METODOLOGÍA CIENTÍFICA Y TEORÍA GENERAL DEL LENGUAJE
}

José Manuel de Cózar Escalante Dpt. de Lógica y $F^{\prime}$ de la Ciencia Universitat de València

Diversos enfoques recientes de la práctica científica, tanto de epistemólogos con sensibilidad social como de sociólogos del conocimiento científico o de la tecnología, coinciden ampliamente en sus críticas hacia las pasadas concepciones positivistas y post-positivistas de la ciencia, presentando en su lugar visiones que, a falta de un rótulo mejor, cabe calificar como 'postmodemas'.

Un grupo de críticas nos interesará aquí en particular por su relevancia: aquellas que hacen depender las justificaciones de la metodología cientufica proporcionadas por el empirismo logico ${ }^{1}$, el post-empirismo kuhniano y el realismo científico de sendas concepciones generales del significado o la referencia. Nuestro propósito es el de proceder a un breve examen de dichas críticas. El fin de dicho examen es el de calibrar la situación en la que se encuentran los estudios de metodología científica por lo que hace a sus vinculaciones con las concepciones sobre el lenguaje existentes ${ }^{2}$.

Como base para exponer las críticas seguiremos a tres autores, cuyos trabajos son de reciente aparición en el campo de la epistemología y metodología científica: Peter Galison, Joseph Rouse y Nancey Murphy ${ }^{3}$.

\footnotetext{
1 Incluidos los enfoques vinculados de un modo u otro a esa tradición, como el de $\mathrm{K}$. Popper y seguidores, a pesar de los intentos efectuados por Popper para desvincularse de sus conocidos del Círculo de Viena.

2 Introducción a la metodología de la ciencia, de Javier Echeverría, incluye un apéndice titulado "Consideraciones sobre una semiología de la ciencia", que aborda con penetración analítica problemas cercanos a los que aquí vamos a tratar.

3 Véanse Galison (1988), Rouse (1991) y Murphy (1990). En lo que sigue, cuando se cite uno de estos autores y una página, ésta corresponderá al trabajo aquí referido.
}

Éndoxa: Series Filosoficas, $n^{\circ}$ 5, 1995, UNED, Madrid:

José $M$. de Cózar Escalante: Metodología cientifica y teoría general del lenguaje. pp. $115-124$. 
En "History, Philosophy, and the Central Metaphor", Galison lanza un duro ataque contra la imagen de la ciencia basada en una jerarquizacion de la tcoría y el experimento que, a su juicio, comparten los positivistas lógicos con sus críticos kuhnianos. A ellos añade Rouse una orientación más reciente, de corte realista científico. Señala Rouse (p. 141) -haciéndose eco de las observaciones de Galison-- que estas tres tradiciones, sucesivamente dominantes en la filosofía de la ciencia, coinciden, a pesar de sus evidentes puntos de conflicto, en ciertos puntos significativos. Para empezar, todas proporcionan una "estructura narrativa" dentro de la que escribir la historia interna de la ciencia; en segundo lugar, asumen que los conceptos metodológicos fundamentales para las ciencias (por ejemplo los de explicación, confirmación y reducción/traducción) son, en importantes aspectos, invariantes a través de las disciplinas científicas y de su desarrollo histórico. También insisten en que hay una relación jerárquica y unitaria (en una u otra dirección) entre la teoría y la observación o experimento. Finalmente, y a juicio de Rouse tal vez lo más importante, las tres tradiciones encuentran en la teoría general del significado y/o la referencia la clave indispensable para la comprensión filosófica de la ciencia.

Como se indicó hace un momento, nos centraremos en este último punto, efectuando un rápido repaso, en orden cronológico, de las tres tradiciones mencionadas: el positivismo logico, el post-empirismo y el realismo científico. Galison vincula el proyecto del Círculo de Viena a una teoría general del lenguaje basada en la suposición de que todo concepto puede ser reducido a un conjunto de proposiciones (el lenguaje protocolario) que reflejan la experiencia sensorial inmediata. Sólo las proposiciones que pudieran expresarse en ese lenguaje o traducirse al mismo tendrían significado. La construcción de un lenguaje universal, un sistema neutral de símbolos, ofrecería la posibilidad de una ciencia unificada, el ideal de los miembros del Círculo de Viena.

Como Galison recuerda, la crítica al positivismo lógico trajo consigo la puesta en cuestión de un lenguaje observacional independiente de la teoría. Primero fue Quine, con su rechazo de los "dogmas" del empirismo. Más tarde, antipositivistas e historiadores de la ciencia (Kuhn, Feyerabend, Hanson, etc.) se complacieron en buscar un ejemplo tras otro 
de la "contaminación" o dependencia de la observación con respecto a la teoría. La concepción general del lenguaje que se ofrecía entonces era una que ponía de manifiesto, a juicio de sus proponentes, la imposibilidad de traducir los lenguajes de los diversos "paradigmas".

Sin embargo, tanto la imagen positivista como la anti-positivista siguen el lenguaje como guía para comprender la evolución científica. Para la primera, la "naturaleza lingǘstica del problema de la ciencia" (Galison, p. 206) se toma patente en la necesidad antes aludida de dar con un lenguaje unitario que sirva como base de reducción de los términos de todas las disciplinas cientficas. Una sola ciencia, desde ese punto de vista, quiere decir una sola estructura lingüística. Los antipositivistas aceptaron estos términos del debate y, consiguientemente, rechazaron la posibilidad de un sola ciencia basándose en la diversidad de "lenguajes" y en la indeterminación de su traducción o "incommensurabilidad del significado". Al no ser posible, en opinión de esos autores, fijar la referencia de los distintos términos desde un sistema lingüístico a otro, la supuesta evolución de la ciencia daba paso a una imagen mucho más discontinua. En pocas palabras (las de Galison, p. 207): "A pesar de sus desacuerdos sobre la existencia de un lenguaje observacional independiente, en el corazón de los enfoques de Kuhn y Camap descansa la suposición de que la actividad de la ciencia ha de ser comprendida principalmente como un desenmarañamiento de las dificultades del lenguaje y de la referencia."

A la exposición de Galison añade Rouse nuevas observaciones sobre positivistas y post-positivistas y sobre todo, hace entrar en escena la tradición realista cientifica que siguió a ambas orientaciones. Por su utilidad para el proposito que nos hemos trazado, recojeremos ahora algunas de esas observaciones (cf. Rouse, pp. 148ss.).

Las primeras conciemen al método científico. Para la filosofía de la ciencia positivista, la ciencia fue identificada con el empleo del método formal. Sus críticos post-positivistas abandonaron en gran medida el formalismo en favor de la autonomía de las disciplinas, pero muchos, como Lakatos, retuvieron una intensa preocupación por salvaguardar la racionalidad metacientífica en la defensa de la metodología y, sobre todo, de una noción altamente general y abstracta de la teoría. Esta proporcio- 
naba el contexto dentro del que los términos teóricos y observacionales adquirían significado. Los primeros post-positivistas, según Rouse, se apoyaron en una teoría general del significado, aliada, via Frege, con la de los positivistas. Pero el post-positivismo temprano fue substituido por el realismo científico ${ }^{4}$. Los realistas de nuevo cuño (R. Boyd o I. Hacking, entre otros) restablecieron aparentemente la conexión entre la representación y la referencia (teorías causales), mirando directamente hacia el mundo como la clave de la comprensión del conocimiento científico. Con todo, justificaron la verdad aproximada de las teorías cientfficas por un criterio de éxito instrumental. En el plano lingüístico, ello correría paralelo a una recuperación de posiciones verificacionistas en el tratamiento de la referencia: emplearon, a juicio de Rouse, un "principio de caridad" para interpretar lo que debía contar como aquello a lo que el lenguaje de los científicos previos se estaba refiriendo. Esa interpretación se haŕa sobre la base de la presente comprensión del interprete (el científico actual) de aquello en lo que consiste el mundo.

Adelantándonos a la parte evaluativa de este trabajo, habría que subrayar lo confuso de este análisis de Rouse cuando afirma que el enfoque verificacionista de la referencia permitió defender un tratamiento realista de la historia de la ciencia. El caso es que el verificacionismo en teoría del lenguaje, tradicionalmente y por talante, no parece casar bien con el realismo, sino más bien al contrario (aunque diversos autores sostuvieran que era neutral en cuestiones ontológicas): si la referencia no se produce por una conexión directa del signo con el objeto, sino por un procedimiento de verificación, cualquiera que éste sea, la conexión entre lenguaje y realidad se torna mucho más indirecta y problemática. Lo que sí resulta más inteligible es el vínculo, mencionado también por Rouse

\footnotetext{
4 ...o, en una interpretación más global del marco de discusión, por el "constructivismo sociológico". Rouse critica este enfoque por preservar, a su entender, la distinción internoexterno en el estudio de la ciencia. No nos vamos a ocupar aquí de esta derivación del examen llevado a cabo por Rouse. En otro orden de cosas, la antología de Boyd et al. (1991) incluye diversos artículos representativos de la reciente polémica en torno al realismo.
} 
(p. 154) entre teorías anti-fregeanas del lenguaje, causales, y realismo cientifico (Kripke, un cierto Putnam).

Sea como fuere, la exposición de Rouse --añadida a la de Galison-resulta clarificadora sobre la actitud de una, por así llamarla, 'filosofía postmoderna de la ciencia' que mete en un mismo saco --el del pensamiento modemo-- todo lo anterior a ella, a saber, las tradiciones positivistas, post-positivistas y del realismo científico. Ateniéndonos a los límites establecidos al comienzo, sólo vamos a evaluar una de las críticas que estos autores postmodernos llevan a cabo: las tres tradiciones dependen de tres concepciones generales del lenguaje, especialmente del significado y la referencia. Esta afirmación no parece ir muy descaminada, pero tampoco constituye una crítica por sí misma. Uno se puede preguntar qué tiene de malo, en principio, vincular entre sí una teoría metodológica y una teoría lingüística con el fin de clarificar el objeto de alguna de ellas o de ambas. 5 . De hecho, las objeciones contra que se ligue el tratamiento del proceder científico con problemas del significado, la referencia, la traducción o cualesquiera otro del lenguaje tiene que ver con una desconfianza típicamente postmoderna hacia toda teoría global $\mathrm{y}$, en concreto, hacia toda teoría lingüística global, especialmente si es representacionalista.

Esta actitud recelosa o incluso de abierto rechazo viene ejemplificada a la perfección, sin necesidad de salimos de nuestro campo, en el tercer trabajo anteriormente aludido, "Scientific Realism and Postmoderm Philosophy" de N. Murphy. La utilidad (y limitación) del enfoque de Murphy reside en la amplitud de términos en los que establece la discusión. Según esta autora --y las tesis de Rouse ya expuestas-- el realismo científico es una posición epistemológica modema. Ahora bien, Murphy ve la modemidad del realismo en que constituye una "aplicacion de una más general teoría representacional del lenguaje a las teorías cientuficas" (p. 300). El motivo de ello es que, siempre según Murphy, el pensamiento modemo se caracteriza en epistemología por ser fundaciona-

Lo cual no quiere decir, por supuesto, que se restrinja el análisis de la naturaleza de la ciencia a la determinación de supuestas necesidades del lenguaje independientes de los resultados y de los métodos de investigación. 
lista, y en filosofia del lenguaje por ser representacionalista (o "referencialista"). Por representacionalismo se entendería la tesis de que "el lenguaje debe obtener su significado primario representando los objetos o hechos a los que se refiere". El pensamiento postmodemo, por su parte, sería holista en epistemología y defendería una teoría del significado como uso en filosofía del lenguaje.

Dejando de lado su patente maniquésmo, de acuerdo con el que los malos (modemos, fundacionalistas y representacionalistas) son finalmente vencidos por los buenos (postmodernos, holistas ${ }^{6}$ y anti-representacionalistas), la dificultad principal de esta manera de abordar el problema reside en que lo simplifica hasta un grado a duras penas sostenible. Ya para comenzar, resulta difícil incluso ponerse de acuerdo con una caracterización de lo postmoderno, dado el maremagnum de variantes que adopta (véase, por ejemplo, Rosenau, 1992). Pero, concediendo algunas simplificaciones por mor de la argumentación que estamos considerando, supongamos que el postmodemismo puede ser caracterizado al menos por contraste con el pensamiento de la modernidad, que sería más fácilmente delineable. Siempre circunscribiéndonos al terreno del lenguaje, cabe, aun a costa de muchos reparos sobre su utilidad, colocar la etiqueta de 'representacionalista' a gran parte de la reflexión moderna sobre el lenguaje. Y también es cierto que con (el segundo) Wittgenstein tiene lugar la revuelta más ambiciosa y contundente contra el representacionalismo moderno de la mente y el lenguaje. También Austin y otros, sin ninguna duda, han contribuido a proporcionamos una imagen del lenguaje más compleja y rica que la anterior, basada en la diversidad de usos. El pensamiento postmodemo gusta de esta imagen fragmentaria, incluso lúdica, del lenguaje.

Un posible lema postmoderno sería "acción en lugar de representación", cuya inspiración habría que buscarla ciertamente en Wittgenstein y Austin. Ese lema se ha extendido, sobre todo por efecto de sociologos

\footnotetext{
6 Aunque colateral con el tema que estamos analizando, cabe mencionar la existencia de una falta de acuerdo entre la tesis de Murphy sobre el carácter distintivamente holista de la epistemología postmoderna (si cabe hablar siquiera de una epistemología postmodema) y el hecho de que Rouse y Galison contemplen a los post-empiristas como holistas pero no, obviamente, como postmodernos.
} 
y etnógrafos de la ciencia (como B. Latour, K. Knorr-Cetina o S. Woolgar), más allá de la pragmática lingǘstica, al mismo estudio de la actividad científica. Debido a todo ello, el acento ha ido desplazándose, a menudo saludablemente, desde un énfasis tal vez exagerado en teorías del lenguaje demasiado omnicomprensivas sobre términos y enunciados (observacionales o teóricos), a la consideración de otros aspectos vinculados a la práctica científica, aspectos tales como la cultura de la instrumentación, las redes comunicativas, las instituciones, los recursos, o cualesquiera otros que puedan resultar de interés. Pero ese desplazamiento no significa que el lenguaje quede en un segundo plano. Antes al contrario, se introduce con mayor fuerza si cabe mediante los tratamientos del texto científico, los símbolos de la instrumentación, la retórica de la ciencia y en el marco más amplio proporcionado por el ya citado concepto de representación ${ }^{7}$.

Por influencia tanto de autores modernos como postmodemos ${ }^{8}$, el interés se ha ido desplazando, paralelamente, desde concepciones metodológicas generales a desarrollos de principios metodológicos para cada dominio y/o tema en concreto (bien se trate de una disciplina científica o una parte de dicha disciplina) ${ }^{9}$. Es todavía algo pronto para saber a ciencia cierta en qué acabará todo ello. Lo que nos interesa recalcar primordialmente en este punto es que las preocupaciones lingüísticas no han desaparecido en absoluto en el estudio de la ciencia, sino que adoptan otra forma, y que esa transformación no hace sino reflejar un significativo cambio en la propia comprensión de la metodología cientifica.

\footnotetext{
7 Mencionaremos, únicamente a título de ejemplo, a Bazerman (1988), Gross (1990), Latour (1987) y Lynch \& Woolgar (eds.)(1990).

8 No obstante, hay que remarcar que los postmodernos tienden a mirar con malos ojos el propio empleo del término 'metodología'.

9 Entre esos autores que ofrecen programas recientes se encuentran L. Laudan, D. Shapere o R. Miller, cultivadores de la filosofía de la ciencia. Rouse (pp. 157ss.) les critica, lo que extiende al propio Galison, el que todos ellos se encuentren todavía demasiado cercanos a la tradición moderna. A juicio de Rouse, estos autores mantienen la idea de que existe una historia intema de la ciencia, y ello les forzaria a basarse, a pesar de sus pretensiones en contra, en una teoría subyacente del significado de corte general.
} 
Respecto a la evaluación de las ya citadas críticas postmodernas sobre la función de las concepciones generales del lenguaje en la comprensión de la metodología cientifica, caben diversas opciones. Veamos un par de ellas.

Como el ataque al representacionalismo trae como consecuencia el rechazo de la concepción usual de la metodología (en sus versiones positivistas o antipositivistas), la primera opción sería la de defender el representacionalismo, en variantes más o menos moderadas, frente a los ataques postmodernos. El cognitivismo (v. Giere, 1988) es --valga el juego de palabras-- el representante actual más destacado de un enfoque representacionalista de la ciencia.

La segunda opción consistira, en cambio, en buscar y explotar posibles incoherencias o contradicciones en la posición anti-modema. Ya quedó señalado que el problema no es en sí la vinculación de visiones generales del lenguaje a determinados enfoques metodológicos, sino el rechazo de dichas teorías generales y de la propia metodología como disciplina. En cuanto a esto último, no cabe colocar en un mismo saco la búsqueda de enfoques más fragmentarios en metodología con la pretensión, mucho más extrema, de convertirla en una "narrativa" y situarla al mismo nivel que otras "narrativas". Tampoco favorecer visiones pragmáticas del lenguaje equivale a una actitud abiertamente escéptica hacia nuestras capacidades lingüísticas. La clave de la posible contradicción sería la siguiente: en su ataque a las teorías generales del lenguaje ino recurre el postmodernismo a una teoría general? ¿No es la teoría del uso una concepción global sobre el lenguaje? En otras palabras, no queda claro si las posturas anti-modemas pretenden substituir la teoría representacional por una concepción del lenguaje basada en el uso o simplemente abandonar toda concepción lingüística general. Y, si optan por lo segundo, resulta menos evidente todavía que sea posible tal abandono.

En realidad, parece más bien que los enfoques postmodemos de la ciencia muestran cierta incoherencia por lo que hace a la cuestión del determinismo lingǘstico (cf. la crítica de Rosenau, 1992, 177ss.). El determinismo lingüístico es la concepción según la cual el lenguaje lo explica todo. En tales instancias el lenguaje se convierte en un mundo en 
sí mismo, constituyendo la esencia de la realidad postmoderna. Toda disciplina es reducida a "narrativa", todo evento a "texto", toda explicación a "interpretación". Fuera del marco lingǘstico no habría nada a lo que poder apelar. Si el lenguaje lo determina todo, entonces los enfoques postmodernos de la metodologia científica están construyendo una jerarquía, atribuyendo una posición privilegiada al lenguaje (y relegando todo lo demás a un plano inferior). Pero, simultáneamente, dichos enfoques subrayan la indeterminación del lenguaje cuando arguyen que hay palabras vacías de contenido, cuando definen el lenguaje como no referencial y no denotacional, sin limitación material. Se advierte que esta perspectiva postmoderna multiplica y manipula el lenguaje de modo que queda desprovisto de todo significado. Las consecuencias de la indeterminación lingüistica son serias. $\mathrm{Si}$ el lenguaje es relativo o arbitrario, entonces nuestras afirmaciones no tienen fundamento. Si lo que se quiere decir con ello es que las palabras no pueden representar verdades garantizadas, pocos disentirán, sean o no postmodernos. Pero los escépticos sugieren que las palabras no tienen significado y que ninguna de ellas puede ser proferida legítimamente. Tendráamos, en suma, una situación como la siguiente, tal como nos la pintan ciertos autores postmodemos: todos los criterios y procederes metodológicos se harían depender de una determinación lingüística inapelable. A la vez, destruida la representación y la referencia, la metodología como tal sería puesta en la picota. Los resultados de los diversos métodos científicos no constiturían conocimiento, ya que no representarian nada, no se referirian a nada fuera del propio lenguaje. En cualquier caso, parece que el enfoque postmoderno de la metodología científica no renuncia a una visión general del lenguaje, siquiera sea para poner en cuestión la propia metodología científica como práctica y como disciplina.

Ahora bien, hay una distorsión injustificable en consideralo todo como texto o narración y asimilar la ciencia a la literatura. Los postmodemos critican con ahínco las teorías generales del lenguaje sólo para hipostasiar a continuación ciertos rasgos del lenguaje y de los signos (por ejemplo la intertextualidad) hasta desvirtuar o negar otros (por ejemplo la referencia). 
$\mathrm{Si}$, en definitiva, los enfoques postmodernos tienen como fin terminar con ciertas ingenuidades y simplificaciones de la metodología tradicional en favor de una visión mas compleja y rica del proceder cientifico, entonces bienvenidos sean. $\mathrm{Si}$, por el contrario y como a menudo parece, suponen más bien la pura negación de todo método, de toda guía positiva de la investigación, entonces poco aportarán al ya de por sí confuso panorama de la reflexión sobre la metodología científica. Mejor es, a nuestro parecer, seguir una vía que, por un lado, integre enfoques representacionales y pragmáticos del lenguaje, y por el otro, contemple el estudio de la metodología basado en la práctica científica dentro de tratamientos metodológicos más globales y equilibrados.

\section{AGRADECIMIENTOS}

Este trabajo se inscribe dentro del proyecto de investigación Lenguaje y mundo (117494/95), subvencionado por la Universitat de València.

Agradezco a Esther Terrón la ayuda que sus comentarios sobre este artículo me han proporcionado.

\section{BIBLIOGRAFIA}

BAZERMAN, C. (1988): Shaping Written Knowledge, University of Wisconsin Press, Madison.

BOYD, R., P. GASPER \& J.D. TROUT (eds.)(1991): The Philosophy of Science, The MIT Press, Cambridge, MA.

ECHEVERRIA, J. (1989): Introducción a la metodología de la ciencia. La filosofía de la ciencia en el siglo $X X$, Barcanova, Barcelona.

GALISON, P. (1988): "History, Philosophy, and the Central Metaphor", Science in Context 2. pp. 197-212.

GIERE, R. (1988): Explaining Science, The University of Chicago Press, Chicago.

GROSS, A. (1990): The Rhetoric of Science, Harvard University Press, Cambridge, MA. LATOUR, B. (1987): Science in Action, Harvard University Press, Cambridge, MA.

LYNCH, M. \& S. WOOLGAR (eds.)(1990): Representation in Scientific Practice, The MTT Press, Cambridge, MA.

MURPHY, N. (1990): "Scientific Realism and Postmodern Philosophy", Brit. J. Phil. Sci., 291-303.

ROSENAU, P.M. (1992): Post-Modernism and the Social Sciences, Princeton University Press, Princeton.

ROUSE, J. (1991): "Philosophy of Science and the Persistent Narratives of Modernity", Stud. Hist. Phil. Sci. Vol. 22, No.1, pp. 141-162. 


\section{LA RETORICA DE LA CIENCIA. ORIGENES Y PERSPECTIVAS DE UN PROYECTO DE ESTUDIO DE LA CIENCIA. ${ }^{1}$}

Javier Gómez Ferri Universitat Jaume I

Entre las corrientes u orientaciones que en estas dos últimas décadas se han incorporado al estudio de la ciencia, la retórica de la ciencia cuenta con un lugar bastante significativo. Otros casos son los de la sociologia del conocimiento científico (SSK), los estudios feministas de la ciencia y lo que se puede denominar "crítica literaria de la ciencia". Hasta el surgimiento de la retórica de la ciencia, a mediados de los años setenta, "retórica" y "ciencia" eran ámbitos completamente separados.

Esa separación todavía sigue existiendo según el punto de vista de muchos estudiosos de la ciencia. Hablar de "retorica de la ciencia" plantea una contradicción que parece obvia, señala Joseph Gusfield (1976) al principio de su artículo "The Literary Rhetoric of Science: Comedy and Pathos in Drinking Driver Research", trabajo que vamos a considerar como el punto de partida del estudio retórico de la ciencia. Está ampliamente asumido que la ciencia versa sobre la verdad, es decir, sobre lo necesario; la retórica, en todo caso, versa sobre lo plausible, es decir, sobre lo contingente. Dicho con palabras de uno de los críticos más hostiles de la retórica de la ciencia: "donde esté la verdad la retórica no tiene cabida" (Munz 1990).

1 Este trabajo ha podido ser realizado gracias a la concesión por parte del Ministerio de Educación y Ciencia de una ayuda para "estancias breves", realizada en la University of Notre Dame. Asimismo quiero agradecerles a Juan Ilerbaig, Vicente Benet y Amador Antón sus críticas y comentarios a este trabajo.

2 Recientemente se ha acuñado la etiqueta "cultural studies of science" para incluir una parte de estos estudios (cf. Rouse 1993). Una presentación sistemática de todas estas corrientes puede encontrarse en Gómez Ferri (1994).

Endoxa: Series Filosoficas, $n^{\circ}$ 5, 1995, UNED, Madrid:

Javier Gomez Ferri: La retórica de la ciencia.Origenes y perspectivas de un proyecto de studio de la ciencia

pp. $125-144$. 
Desde este punto de vista, el único papel que podría asumir la retórica en el ámbito de la ciencia sería un papel negativo. La retórica de la ciencia sería una "retórica del error", cuya tarea consistiría en dar cuenta de por qué ciertas creencias falsas o fraudulentas son aceptadas y tomadas por verdaderas o científicas durante un cierto periodo de tiempo. Dicha concepción deriva claramente de la tradición platónica, en la cual la retórica tiene que ver con el engaño mediante el discurso. Frente a esa concepción negativa, aquí quiero tratar de la retórica como proyecto de investigación y estudio de la argumentación científica en general.

El antecedente directo de la aplicación de la retórica al estudio de la ciencia es un movimiento de renovación, surgido en la década de 1950 , la "nueva retórica." Además de este movimiento, el surgimiento de la retórica de la ciencia también ha estado influido por el declive de un cierto estilo de hacer filosofía de la ciencia, aquél que se ha ocupado principalmente del análisis de la estructura lógica de las teorías científicas. Antes de tratar de la retórica de la ciencia realizaré un breve esbozo histórico que ayude a entender las razones de la diversidad actual que caracteriza dicho proyecto y sirva, al mismo tiempo, para comprender sus potencialidades y sus limitaciones.

\section{La Retórica}

La Retórica cuenta con un gran, aunque difícil, pasado; un pasado de casi 2500 años. Sus orfgenes los encontramos en la cultura y en el pensamiento griegos del siglo $\mathrm{V}$ a. C. La Retórica nace ligada al mundo de la política, concretamente a la democracia como sistema participativo de gobiemo. La primera orientación o corriente filosofica con la que la retórica está relacionada es la Sofística. Las necesidades planteadas por la práctica democrática en la polis griega llevaron a los sofistas a tenerla como uno de los elementos principales en sus enseñanzas. Después de ellos, Platón relegaría la retórica a un plano muy secundario, al separar el ámbito de la opinión (doxa) del de la ciencia o el conocimiento verdadero (episteme). Para él, la retórica consistía fundamentalmente en el arte de encantar el alma con palabras (Fedro, 261a). Con Platón se abría un abismo entre retórica y ciencia que sólo recientemente está 
empezando a ser salvado. Aristóteles,por su parte, no rompió la separación que introdujo su maestro, pero sí contribuyó a dignificar la retórica. La retórica y la dialéctica se repartirían el ámbito de verdades no sujetas a demostración apodíctica. Esto es, se repartirían el ámbito de las verdades comunes o contingentes. Aristóteles fue, además, el primero en ofrecer una sistematización de la retórica. Tras el Estagirita, la retórica inició una progresiva salida del mundo filosófico hasta que, en la modernidad, su ámbito propio fue el de la creación literaria.

En todo ese lapso tiempo son muchas las contribuciones que se pueden mencionar. Entre todas ellas cabe destacar las de Cicerón y Quintiliano, los máximos cultivadores de la retórica en el mundo latino. Para Cicerón la retorica tendría que ver con el arte de hablar o hacer discursos públicos; para Quintiliano, con el arte del bien decir. Durante la Edad Media la situación de la retórica se mantuvo más o menos estacionaria. En ese periodo la retórica quedó definitivamente integrada en el trivium, junto a la gramática y la dialéctica. En el Renacimiento, el interés por la retórica resurgió. Sin embargo, a medida que la concepción modema del conocimiento se fue implantando, la retórica fue perdiendo terreno. Ya con Petrus Ramus, aún en el Renacimiento, se inicia la tendencia moderna a considerar la retórica como el arte de la elocuencia y el omato, dejando a la lógica el ámbito del conocimiento. Tras Ramus, pensadores como Bacon, Descartes, Locke o Leibniz insistirian que el conocimiento es conocimiento demostrado a partir de principios indubitables y autoevidentes. Una de las acusaciones más duras contra la retórica la podemos encontrar en las siguientes líneas del Ensayo sobre el Entendimiento Humano de John Locke:

Si pretendemos hablar de las cosas como son, es preciso admitir que todo el arte de la retórica, exceptuando el orden y la claridad, todas las aplicaciones artificiosas y figuradas de las palabras que ha inventado la elocuencia, no sirven sino para insinuar ideas erróneas, mover las pasiones y equivocar así el juicio, de manera que en verdad no es sino superchería. $Y$ por lo tanto [...] cuando se trate de la verdad y del conocimiento, no pueden menos de tenerse por gran falta, ya sea del lenguaje, ya de la persona que las emplea (Locke, Lib. 3, Cap. X, § 34). 
Aunque los argumentos filosóficos en contra tuvieron un peso importante, la crisis de la retórica clásica estuvo provocada también por otras causas. Dos hechos que condicionaron la función y el posterior destino de la retórica fueron, por un lado, la escasa relevancia del orador en el mundo moderno, en comparación con el mundo grecorromano, $y$, por otro, el creciente papel del texto escrito, propiciado por la creación de la imprenta. Así las cosas, la retórica quedaría, en la modernidad, relegada al ámbito literario, asumiendo el papel de la poética y ocupándose del estudio de la creación literaria. Esa situación se mantendría hasta mediados del siglo XX, momento en el que surge lo que se conoce como "nueva retórica." Este movimiento de renovación orientará la retórica en una nueva dirección, lejos de la estrictamente literaria. Con la "nueva retórica," según lo expone Mortara Garavelli (1988:25), la retórica queda escindida, por un lado, en "teoría de las figuras" y, por otro, en "teoría de la argumentación". Esta escisión va a ser propicia para el surgimiento de la "retórica de la ciencia".

A pesar de la importancia que la "nueva retórica" tiene en la renovación del pensamiento sobre el lenguaje argumentativo, no todos los méritos deben ser atribuidos al mencionado movimiento. Conviene tener presente que desde la década de 1930 el interés por el estudio del lenguaje fue una cuestión central para varias disciplinas. Entre ellas cabe destacar tanto la filosofía como la teoría de la comunicación. Con respecto a la primera, qué duda cabe de que un movimiento filosófico como el positivismo lógico alentó sobremanera, desde su particular posición, el estudio del lenguaje (Perelman 1990:807). Un estudio que se extendería posteriormente a los aspectos prácticos del lenguaje en lo que se ha dado en llamar filosofía del "lenguaje ordinario". La filosofía del "lenguaje ordinario", así como también el pragmatismo filosófico americano, constituyeron un caldo de cultivo muy propicio para el desarrollo de la retórica contemporánea. 
Finalmente, conviene hacer mención también de las contribuciones realizadas por I. A. Richards (1936) o Kenneth Burke (1950) desde la propia retórica. Estos dos autores, aparte de recuperar el estudio de la retórica, supieron percibir el alcance que para ésta iba a tener un nuevo fenómeno en la cultura occidental, "la sociedad de masas", fenómeno propiciado por la aparición en la década de 1930 de las nuevas tecnologías de la comunicación o mass-media.

\section{La nueva retórica}

Como he anticipado, la rehabilitación definitiva de la retórica se produce a finales de los años cincuenta con el surgimiento de la "nueva retórica." En 1958 fueron publicados dos trabajos que, aunque son distintos en su concepción, son similares en los propósitos y en la dirección hacia la que apuntan. Esos trabajos son, respectivamente, el de Chaïm Perelman y Lucie Olbrechts-Tyteca, Traité de l'Argumentation, y el de Stephen Toulmin, The Uses of Argument. Son Perelman y Olbrechts-Tyteca quienes hacen uso de la denominación "nueva retórica". Ambos encuentran sus raíces en Aristóteles, a quien consideran el padre de la teoría de la argumentación. Alejándose de la concepción de la retórica como disciplina dedicada al estudio del ornato lingüístico, Perelman y Olbrechts-Tyteca (1958:34) definen ésta como "el estudio de las técnicas discursivas que permiten provocar 0 aumentar la adhesión de las personas a las tesis presentadas para su asentimiento".

Tanto Perelman y Olbrechts-Tyteca como, en su caso, Toulmin vienen a insistir en una idea básica: las posibilidades de la argumentación y el conocimiento racional son variadas, no quedando exclusivamente reducidas al conocimiento y a la argumentación científicas. Entre los extremos del conocimiento demostrado, conocimiento que hay que aceptar por necesidad, y la ignorancia y la irracionalidad, el conocimiento y la argumentación 
se dan en una amplia gama de posibilidades. Y es que el campo de la argumentación es el de lo verosímil y lo plausible, no el de lo necesario, advierten Perelman y Olbrechts-Tyteca (1958:30). En consonancia con esta idea, Perelman, Olbrechts-Tyteca consideran inadecuada la lógica formal como instrumento de análisis de la argumentación en el ámbito cotidiano, jurídico o moral, e incluso para algunas ciencias sociales. Consideran estos autores que "justificar" es dar buenas razones para creer en algo, y no necesariamente demostrar formalmente ese algo. Ambos autores estaban, con sus ideas y propuestas, abriendo nuevas perspectivas sobre la racionalidad.

La publicación de un tratado dedicado a la argumentación y su vinculación a una antigua tradición, la de la retórica y la dialéctica griegas, constituyen una ruptura con la concepción de la razón y del razonamiento que tuvo su origen en Descartes y que ha marcado con su sello la filosofía occidental de los tres últimos siglos [...] Fue Descartes quien, haciendo de la evidencia el signo de la razón, sólo quiso considerar racionales las demostraciones que, partiendo de ideas claras y distintas, propagaban, con ayuda de pruebas apodícticas, la evidencia de los axiomas a todos los teoremas (Perelman y Olbrechts-Tyteca 1958:31).

Toulmin (1958) no está lejos de los puntos de vista de Perelman y Olbrechts-Tyteca. Coincide con esos dos autores cuando señala que la argumentación lógica ocupa un papel marginal tanto en la vida como en la ciencias. $\mathrm{Y}$ con ellos comparte también el reproche al ideal matemático o silogístico del conocimiento que ha monopolizado la epistemología durante la modernidad. Ello no quiere decir que los autores mencionados pretendan prescindir de la lógica o negar su validez. De lo que tratan es de señalar sus limitaciones y, en todo caso, de complementarla.

A pesar de lo que se acaba de exponer, debe tenerse muy presente que la "nueva retórica" no es una "retórica de la ciencia". 
El ámbito de aplicación de aquélla está restringido a la filosofía, el derecho $\mathrm{y}$, a lo sumo, a algunas de las ciencias humanas (Overington 1977:154). Es en la segunda mitad de la década de 1970 cuando toda la ciencia va a convertirse en objeto de análisis de la retórica. Ese es. el momento en que puede hablarse propiamente de "retórica de la ciencia".

\section{La retórica de la ciencia}

La ciencia es, probablemente, el ámbito por antonomasia de la persuasión y de la argumentación. Esos dos parecen ser los objetos propios de estudio de la retórica. Sin embargo, como ya he señalado, "retórica" y "ciencia" han sido dos ámbitos que han permanecido separados hasta hace muy poco tiempo. En el seno de la tradición intelectual occidental, la retórica ha sido menospreciada y relegada a un segundo plano, y muy particularmente lo ha sido en relación con la ciencia.

No obstante, la arraigada separación entre retórica y ciencia no cabe atribuirla únicamente a la existencia de un rechazo ancestral hacia la primera. Hay que tener presente, advierte Bazerman (1988:322), que los objetivos, el contexto y las herramientas de la retórica política del ágora de la polis griega y los de la argumentación científica moderna son sustancialmente distintos, tan distintos que no resulta fácil la aplicación de las categorías de la retórica clásica al estudio de la ciencia. Asimismo, la tendencia a idealizar excesivamente la racionalidad y el lenguaje científicos no ha facilitado el acercamiento entre retórica y ciencia.

La consideración del lenguaje de la ciencia como un vehículo neutral, como un simple instrumento de descripción de la realidad -postura mantenida desde comienzos de la modernidad y reforzada por el positivismo lógico a comienzos del siglo XX- ha hecho muy difícil el estudio retórico y literario de la ciencia. La fijación por un lenguaje y una gramática ideales a los que la ciencia ha de 
responder ha pesado fuertemente en esta dirección. ${ }^{3}$ Como bien apunta Bazerman (1988), la ciencia ha sido tenida por un código simbólico privilegiado, bien gracias a que se le ha atribuido una especial relación con la realidad, o bien a su alianza con las matemáticas. Desde esta perspectiva, no resulta difícil pensar que la retórica o, en su caso, la teoría literaria poco o nada podían aportar al estudio del lenguaje científico.

En la actualidad la retórica de la ciencia no se caracteriza por ser una corriente excesivamente homogénea y bien definida. Tiene lazos diversos, los cuales van desde la filosofía hasta la sociología, pasando por la crítica literaria. No obstante, a pesar de esa diversidad, los retóricos de la ciencia comparten una idea central: su objeto de estudio es la persuasión y la argumentación científica. Entre las tareas de los científicos está la de comunicación de sus investigaciones. Pero los científicos no se limitan simplemente a comunicar sus resultados, sino que también tratan de convencer a otros científicos para que los acepten. Para ello hacen uso de unos determinados recursos expresivos, y es esto lo que confiere carta de legitimidad a la retórica de la ciencia.

Junto a ello, los retóricos de la ciencia comparten también el punto de vista de que gran parte de las argumentaciones, entre ellas las científicas, no son sensu stricto lógico-formales, aunque $a$ posteriori sean analizables con un instrumental lógico. Así lo expresa Maurice Finocchiaro en su artículo "Logic and Rhetoric in Lavoisier's Sealed Note: Toward A Rhetoric of Science", cuando afirma que

[1]as consideraciones puramente lógicas, las argumentaciones racionales y las llamadas a las reglas del método científico no

3 Sobre la distinta consideración del lenguaje científico desde la metodología de la ciencia y desde otras perspectivas como la retórica de la ciencia, la sociología del conocimiento científico y la teoría literaria puede verse Gómez Ferri (1995). 
son suficientes para hacer que los científicos cambien de ideas (Finocchiaro 1977:111).

Este hecho viene a reafirmar el papel de la retórica de la ciencia. Más recientemente, hay quienes, como Lawrence Prelli (1989), han ido más lejos y no sólo han puesto en duda que los científicos se guíen exclusivamente por criterios lógicos y empíricos a la hora de elegir unas teorías frente a otras, sino que ni siquiera tales criterios son suficientes para reconstruir racionalmente las teorías científicas. Esa insuficienciano sólo hace factible, sino también necesario un proyecto de estudio retórico de la ciencia

Científicos y estudiosos de la ciencia han sido cada vez más conscientes de que las pruebas apodícticas son raras, incluso en la ciencia, y que los conceptos asociados con la lógica formal son insuficientes para describir las actividades del 'quehacer científico'. Ellos han comenzado, por tanto, a reconsiderar cómo tratar y pensar la actividad científica (Prelli 1989:1).

En lo que hemos visto puede comprobarse que existe una cierta similitud entre los puntos de vista de la "nueva retórica" y los de la retórica de la ciencia. Sin embargo, con la retórica de la ciencia se introduce una novedad importante. La ciencia va a dejar de ser considerada un ámbito especial o excepcional de argumentación y de discurso. Como hemos visto, para Perelman y Olbrechts-Tyteca las ciencias naturales y las matemáticas quedaban excluidas del análisis retórico. Tampoco Toulmin estaba pensando expresamente en la ciencia cuando escribió The Uses of Argument. ${ }^{4}$ Así pues,

4 Esta posición es comparable con la que se da dentro de la sociologia del conocimiento. Karl Mannheim, el fundador de ésta, excluyó a las ciencias naturales y a las matemáticas del análisis sociológico. No fue hasta mediados de la década de los setenta. con la aparición de la sociología del conocimiento científico (SSK), cuando se consideró que cualquier tipo de conocimiento era susceptible de ser estudiado sociológicamente. Cada vez son publicados en castellano un mayor número de trabajos sobre la sociología del conocimiento científico. Uno reciente y muy recomendable es el de Emilio Lamo de Espinosa, Jose María González y Cristobal Torres (1994). 
lo que hace la retórica de la ciencia es considerar que la ciencia no escapa al análisis retórico.

El principal impulso para dicha ampliación le llega a la retórica desde fuera de esa misma disciplina. Concretamente le va a venir desde parte de la filosofía antipositivista de la ciencia realizada en las décadas de 1960 y 1970. Las ideas de Thomas S. Kuhn, Michael Polanyi, Norwood R. Hanson, Paul Feyerabend y John Ziman, entre otros, ejercieron una influencia importante para que se diera el paso que, desde la retórica, conduciría al nacimiento de la retórica de la ciencia.

De entre todos estos autores, ha sido Kuhn el que ha ejercido una influencia más significativa en relación con el proyecto de estudiar la ciencia desde un punto de vista retórico..$^{5}$ La concepción de Kuhn sobre la dinámica del saber científico abrió la posibilidad de estudios de la ciencia con una orientación distinta a la tradicional, la propiamente filosófica. Al defender, en $L a$ estructura de las revoluciones científicas, que el cambio de un paradigma científico a otro puede deberse a "razones que no se derivan de la estructura lógica del conocimiento científico" (1962:153), Kuhn daba pie a que los factores sociales, retóricos, estéticos o de otra índole fueran considerados relevantes en el estudio de la ciencia y de su dinámica.

Con los antecedentes que ya hemos visto, y en un contexto como éste, poco tardaría en surgir la retórica de la ciencia. Si tuviéramos que fijar un año para señalar el comienzo de ésta, ese año sería el de 1976. En esa fecha aparece publicado "The Literary Rhetoric of Science: Comedy and Pathos in Drinking Driver Research", de Joseph Gusfield. Aunque ciertamente existen

5 Ello no quiere decir que se le deba atribuir una especial paternidad a Kuhn con respecto a la retórica de la ciencia. Esta misma cuestión, en cambio, no está clara con respecto a la sociología del conocimiento científico. Dos posturas sobre el particular pueden verse en Barnes (1982) y Pinch (1990). 
otros trabajos retóricos sobre la ciencia anteriores al de Gusfield, sólo en éste encontramos una especial percepción de la existencia de un área de investigación, superando la vieja dicotomía entre ciencia y retórica. Además, su artículo no fue el único que apareció en 1976 sobre el tema que nos ocupa. Ese año, Philip C. Wander publicó un trabajo, no muy relevante en lo que respecta a su contenido, pero cuyo título era muy significativo: "The Rhetoric of Science."

Justo al año siguiente, en 1977 , nuevas aportaciones vinieron a sumarse a las anteriores, como son: "Science as a Rhetorical Transaction: Toward a Nonjustificational Conception of Rhetoric", de W. B. Weimer; "Logic and Rhetoric in Lavoisier's Sealed Note: Toward A Rhetoric of Science", de M. A. Finocchiaro; "The Scientific Community as Audience: Toward a Rhetorical Analysis of Science", de M. A. Overington; y "La Rhétorique de la Science", de B. Latour y P. Fabri. Este caudal de trabajos puso de manifiesto que la retórica de la ciencia era algo más que un mero fenómeno episódico. Sin embargo, la consolidación plena de esta corriente de estudio de la ciencia a finales de la década de los ochenta.

El artículo de Gusfield, al que ya me he referido, es un análisis retórico sobre determinados estudios sociológicos en tomo a la conducción bajo los efectos del alcohol. Lo que Gusfield trataba de mostrar con este trabajo era el poder conformador del lenguaje, en este caso del lenguaje científico, con respecto a la realidad. Contrariamente a lo que habitualmente se piensa del lenguaje de la ciencia, los objetos $\mathrm{y}$ hechos descritos o explicados no son totalmente independientes del lenguaje empleado (Gusfield 1976:16). Gusfield considera que el lenguaje científico no es un mero medio o instrumento que simplemente nos informa acerca de la realidad. Para Gusfield, el lenguaje científico posee una componente expresiva de la que aquél no puede desligarse. No es, pues, un instrumento neutral de transmisión de contenidos o de 
representación de la realidad. Y pretender lo contrario es probablemente la mejor argucia retórica que hasta ahora se ha inventado.

Gusfield (1976) trató de mostrar que a los trabajos por él analizados subyacía una determinada política, la cual se dejaba entrever a través de la caracterización que se hacía de dicha problemática. Gusfield, señala Alan Gross, puso de manifiesto que

[l]a propuesta política que se seguía de las investigaciones sobre conductores ebrios no derivaba de los datos; más bien éstos fueron adecuados a los intereses del investigador, con el fin de convertir un ciudadano irresponsable en una criatura patética dominada por la enfermedad, un problema legal en un problema médico y psiquiátrico (Gross 1990b:341).

Para Gross (1990a:49), el trabajo pionero de Gusfield, sin embargo, todavía está preso de una cierta concepción negativa de "retórica", aquélla "retórica del error" a la que nos referíamos al comienzo de este artículo. ${ }^{6}$ Esa componente negativa irá desapareciendo a medida que vaya consolidándose el proyecto de estudio retórico de la ciencia.

Durante la primera década de vida de la retórica de la ciencia casi todos los trabajos publicados son artículos. Prácticamente no aparece ni un solo libro de retórica de la ciencia antes de 1985. A partir de ese año, sin embargo, se va producir una verdadera explosión de libros de o sobre la retórica de la ciencia. En 1985, el economista Donald McCloskey publica The Rhetoric of Economics (1985), un análisis retórico de la ciencia aplicado a la economía. ${ }^{7} \mathrm{Al}$ año siguiente John A. Schuster y Richard R. Yeo

Según Gross (1990a), Gusfield sigue fiel a la idea aristotélica de la superioridad de lo nomológico sobre lo hermenéutico. No obstante la crítica de Gross, hay que tener presente que uno de los puntos centrales del trabajo de Gusfield es la crítica al ideal representacional del lenguaje cientúfico, según el cual éste es como una "cristalera" a través de la cual el mundo se nos manifiesta tal y como verdaderamente es (Gusfield 1976:16).

7 De la retórica de la economía me ocupo detalladamente en otro trabajo, "La retórica de la economía. Un análisis crítico" (manuscrito). 
editan The Politics and Rhetoric of Scientific Method. El libro de Schuster y Yeo, sin embargo, apenas trata de los aspectos discursivos, sino que más bien se centra en la cuestión del poder retórico que supone la apelación a lo científico. A partir de ese año la publicación de trabajos aumenta tanto cuantitativa como cualitativamente, quedando así constituida la retórica de la ciencia como una nueva área de estudios sobre la ciencia. ${ }^{8}$

Como ya he señalado, la retórica de la ciencia es en estos momentos más un movimiento o corriente que una disciplina bien definida. Su punto de partida es considerar que la ciencia es el

8 Sin tratar de ser exhaustivo, mencionaré los más representativos. En 1987, John Nelson, Alan Megill y el propio McCloskey editan un conjunto de trabajos sobre la retórica de las ciencias humanas, The Rhetoric of the Human Sciences (1987). Dicho libro es fruto de un congreso celebrado en la Universidad de Iowa en 1984. El propósito de dicho congreso era reivindicar la retórica y convertirla en un medio de investigación científica. En 1988 Arjo Klamer, Donald McCloskey y Robert Solow editan The Consequences of Economic Rhetoric, resultado de un congreso celebrado en 1986 en tomo a la retórica de la economía. El libro es desigual, pero sirve para mostrar que hay varias concepciones de retórica. Ese mismo año Bazerman publica Shaping Written Knowledge (1988), libro que ha sido señalado como un trabajo modélico dentro del análisis literario de la ciencia. El libro de Bazerman, que recoge trabajos realizados por este autor durante casi una década, conjuga perfectamente el estudio retórico, literario y sociológico de la ciencia. A finales de la década, en 1989 encontramos el primer intento de ofrecer una sistematización de la disciplina con el libro A Rhetoric of Science de Lawrence J. Prelli. El trabajo de Prelli constituye una reivindicación del estudio de la ciencia basado principalmente en la retórica clásica, sobre todo en las categorias de los stasis y los topoi. Ese año, Herbert. W. Simons, autor de otros libros sobre retórica, edita The Rhetoric in the Human Sciences (1989) y The Rhetorical Turn (1990), al año siguiente. Ambos volúmenes son resultado de un congreso celebrado en 1986 en la Universidad de Temple -según su organizador, una secuela del congreso de Iowa de 1984 del que ya he hecho mención. En 1990 Greg Myers y Alan Gross publican, respectivamente, dos excelentes trabajos sobre retórica de la ciencia. Writing Biology (1990) de Myers es un libro dedicado al estudio de publicaciones científicas en la Biología y se halla a caballo entre el análisis social del discurso científico y la retórica de la ciencia. El de Gross, The Rhetoric of Science (1990), es uno de los trabajos donde se defiende una de las perspectivas más radicales dentro de la retórica de la ciencia. Gross lleva sus propuestas hasta posiciones anti-realistas. Mucho más tradicionales son los trabajos que están recogidos en el libro editado por Marcello Pera y William R. Shea, Persuading Science. The Art of Scientific Rhetoric (1990). Finalmente, y para concluir esta enumeración (de la cual he excluido los trabajos de los sociólogos del conocimiento científico dedicados al análisis del discurso científico) cabe mencionar el reciente trabajo del filósofo de la ciencia, Steve Fuller, Philosophy, Rhetoric and the End of Knowledge (1993). Desafortunadamente el contenido del libro hace poco honor a las expectativas que su título hace albergar. 
fenómeno persuasivo por excelencia dentro de nuestra cultura. Y se ve legitimada por el hecho de que los aspectos lógicos y empíricos no bastan para explicar por qué unas teorías son aceptadas en lugar de otras. En relación con esta cuestión, dos de los puntos más duramente criticados por los retóricos de la ciencia han sido el fundamentalismo epistemológico y el ideal metodológico del conocimiento, puntos que subyacen a buena parte de los estudios sobre la ciencia, sobre todos a lo de índole filosófica. Más allá de esos puntos resulta difícil dar unas características concretas que valgan para todos aquellos autores que forman parte de la retórica de la ciencia. A pesar de ello, he intentado, en lo que sigue, sistematizar mínimamente el panorama de dicha disciplina alrededor de varias cuestiones como son la metodología, la epistemología y la tradición o disciplina por la que están más influidos (Véase infra cuadro 1).

Por su actitud respecto al análisis metodológico de la ciencia, podemos distinguir entre quienes defienden el estudio retórico de la ciencia como un enfoque complementario y quienes lo adoptan como un enfoque sustitutivo. Los primeros, entre los que destacan autores como Finocchiaro, Bazerman o Klamer, plantean la retórica como un complemento que llega donde no puede llegar otro tipo de estudios como son la lógica y la metodología de la investigación científica. Sin negar la validez del método científico, pretenden poner de manifiesto que la racionalidad científica no puede reducirse a un conjunto de reglas o normas metodológicas. Desde esta perspectiva, la retórica puede formar parte del estudio metodológico de la ciencia. Los segundos, entre los que se hallan, por ejemplo, Gross o McCloskey, dudan de la viabilidad de la metodología de la ciencia o simplemente la rechazan de plano y, por tanto, ven la retórica de la ciencia como el tipo de estudio destinado a sustituir o reemplazar a aquélla. Para ellos, las apelaciones basadas en el método científico son propiamente retóricas. (Véase cuadro, infra). 


\begin{tabular}{||l|l|l|l||}
\hline $\begin{array}{l}\text { Retórica de la } \\
\text { Ciencia }\end{array}$ & Metodología & $\begin{array}{l}\text { Epistemolo- } \\
\text { gía }\end{array}$ & Tradición \\
\hline Gusfield (1976) & $\begin{array}{l}\text { Complemen- } \\
\text { tario }\end{array}$ & Realista & $\begin{array}{l}\text { Teorfa litera- } \\
\text { ria }\end{array}$ \\
\hline Finocchiaro (1977) & $\begin{array}{l}\text { Complementa- } \\
\text { rio }\end{array}$ & Realista & Filosofía \\
\hline Prelli (1990) & Sustitutivo & Realista & $\begin{array}{l}\text { Teorfa de las } \\
\text { figuras }\end{array}$ \\
\hline $\begin{array}{l}\text { Klamer (1983, } \\
\text { 1987) }\end{array}$ & $\begin{array}{l}\text { Complementa- } \\
\text { rio }\end{array}$ & Realista & $\begin{array}{l}\text { Teoría de la } \\
\text { argumentación }\end{array}$ \\
\hline Bazerman (1988) & Complementario & Realista & $\begin{array}{l}\text { SSK/Teoría } \\
\text { literaria }\end{array}$ \\
\hline McCloskey (1985) & Sustitutivo & Anti-realista & $\begin{array}{l}\text { Teoría de las } \\
\text { figuras }\end{array}$ \\
\hline Gross (1990 & ^Sustitutivo & Anti-realista & ssK \\
\hline
\end{tabular}

Asociadas a las cuestiones concernientes a la metodología están las de índole epistemológica. Suele darse una correlación entre las posiciones mantenidas en uno y otro ámbito, aunque no en todos los casos tiene por qué ser así. Dentro de un mismo ambiente anti-fundamentalista, hallamos a quienes pretenden conjugar realismo y retórica, como son los casos de Bazerman o Prelli, o también a los más radicales, como McCloskey o Gross, que mantienen una posición anti-realista. (Véase cuadro 1, supra).

Finalmente, un tercer tema que nos puede ayudar a situar buena parte de los estudios de la retórica de la ciencia es la disciplina o tradición hacia la que se orientan o en la que se basan (véase cuadro 1). Dado que la retórica de la ciencia se ha constituido recientemente, es frecuente encontrar que los estudios retóricos de la ciencia están influidos por tradiciones, a veces, muy dispares. Los modelos más seguidos son la retórica clásica y los estudios sociales del conocimiento cient́fico. 
Ligados a la tradición clásica están, por ejemplo, los trabajos de Prelli o McCloskey. En el otro extremo se hallarían los de Gross o Bazerman. Ambos extremos entrañan sus peligros. Lo que suele pasar con quienes siguen la perspectiva clásica de la retórica es que acostumbran a olvidar que la ciencia no es simplemente discurso y persuasion, y reducen el estudio de ésta a un análisis de las figuras retóricas. Es lo que sucede, por ejemplo, con McCloskey o Prelli. Para ellos toda la ciencia es retórica. Pero eso constituye un reduccionismo inadmisible. La práctica científica incluye otros elementos distintos del discurso que son difícilmente analizables desde en punto de vista retórico. Ése es un peligro en que pueden caer los retóricos de la ciencia. Sin embargo, no es ése el único problema del que deben ser conscientes. Incluso aunque la ciencia fuera sólo discurso, necesitariamos estudiar ese discurso en su contexto. Por esta razón, muchos de los autores que comienzan por la retórica de la ciencia se ven necesariamente abocados al estudio social del discurso cientifico, como ha sido el caso de Greg Myers (1990b). Indica Myers, por ejemplo, que para entender el discurso científico, entre otras cosas:

Hay que atender a la manera en la que se forman los cientfficos, las estructuras de sus carreras, la manera en que los cientificos hablan en el laboratorio y la manera en la que usan su instrumental, las maneras como consiguen subvenciones y son evaluados, las maneras como escriben, reescriben y leen un artículo (Myers 1990b:562).

En consecuencia, una de las dificultades más grandes a las que se enfrenta la retórica de la ciencia es la posible pérdida de su propia identidad. En este sentido, los estudios retóricos pueden acabar convertidos en una parte de los estudios sociológicos del conocimiento cientffico. Así pues, por un lado, encontramos que la retórica ha de afrontar el peligro de convertirse en un estudio irrelevante, centrado en el estudio de las figuras o tropos lingüísticos; y, por el otro, vemos que corre riesgo de disolverse en otras disciplinas. Entre estos dos extremos cabe una tercera postura, centrada fundamentalmente en los fenómenos de la argumentación y la comunicación: la retórica como estudio de la 
argumentación cientifica. Dirección en la que la mayor parte de la labor está todavía por hacer.'

A pesar de sus pocos años de existencia, el análisis retórico de la ciencia ha abierto nuevas perspectivas sobre el fenómeno científico y ha mostrado que tiene un lugar dentro del ámbito de los estudios sobre la ciencia. A los cientificos les ha hecho ver la riqueza de recursos expresivos con la que cuentan; a los estudiosos de la ciencia les ha abierto los ojos en torno a la argumentación cientifica. Pero, sobre todo, la retorica de la ciencia ha aportado cierta luz sobre la propia racionalidad cientufica, al mostrar que ésta es algo más amplio de lo que la teoría de la ciencia tradicional habia hecho ver. Cualquier temor que pudiera generarse ante esta situación es completamente infundado. Desde la retórica no se cuestiona ni la ciencia ni la racionalidad cientffica, lo que se cuestiona son puntos de vista arraigados en algunas de las disciplinas que tradicionalmente se han dedicado al estudio de una y otra. Unos puntos de vista que tienden más a idealizar la ciencia que a acercarse a la realidad de su propia práctica.

9 No todos los estudios sobre retórica de la ciencia caben en los ejes que acabo de trazar. Así, por ejemplo, muchos de esos estudios podrian ser sencillamente considerados estudios de filosofía de la ciencia, mientras que otros bien podrian caer dentro de la teoría o crítica literaria, como es el caso de los análisis que recurren al estructuralismo y al postestructuralismo. Estos últimos se centran, sobre todo, en las cuestiones textuales. Ya he señalado que en este caso se podía hablar de "crítica literaria de la ciencia", o también de "estudios culturales de la ciencia" (cf. Rouse 1993). Con respecto a los análisis sociales del discurso científico practicados por sociólogos del conocimiento científico como G. Nigel Gilbert y Michel Mulkay (1984), simplemente advertir que no considero que los análisis de éstos sean propiamente análisis que caigan fácilmente dentro de la etiqueta "retórica de la ciencia", a pesar de la existencia de algunas similitudes. Por un lado, retórica y sociología del conocimiento nacen de dos tradiciones muy distintas. Por otro, la importancia que se otorga al contexto del discurso es muy'distinta en uno y otro caso. 


\section{REFERENCIAS BIBLIOGRAFICAS}

BARNES, Barty, (1982), T. S. Kuhn y las ciencias sociales. F.C.E., México, 1986.

BAZERMAN, Charles (1988), Shaping Written Knowledge. The Genre and Activity of the Experimental Article in Science. University of Wisconsin Press, Madison.

BURKE, Kenneth (1950), A Rhetoric of Motives. California University Press, Berkeley.

FINOCCHIARO, Maurice A. (1977), 'Logic and Rhetoric in Lavoisier's Sealed Note: Toward A Rhetoric of Science', Philosophy and Rhetoric, 10(2):111-122.

FULLER, Steve (1993), Philosophy, Rhetoric and the End of Knowledge. The Coming of Science and Technology Studies. University of Wisconsin Press, Madison.

GILBERT, G. Nigel y Michel MULKAY (1984), Opening Pandora's Box. A sociological analysis of scientists' discourse. Cambridge University Press, Cambridge.

GOMEZ FERRI, Javier (1994), Metodología de la economía: El enfoque pragmático. (Tesis Doctoral. Mictoficha). Publicaciones de la Universitat Jaume I, Castellón.

GOMEZ FERRI, Javier (1995), 'La ciencia como relato. Hacia una post-metodología de la ciencia', en Vicente Sánchez Biosca y Rafael Rodriguez Tranche (eds.) (1995), La crisis del relato en la contemporaneidad. Filmoteca de Andalucía, Córdoba (en prensa).

GROSS, Alan G. (1990a), The Rhetoric of Science. Harvard University Press, Cambridge, MA.

GROSS, Alan G. (1990b), 'An English Professor Looks at the Scientific Article', Studies in the History and Philosophy of Science, 21(2):341-349.

GUSFIELD, Joseph (1976), 'The Literary Rhetoric of Science: Comedy and Pathos in Drinking Driver Research', American Sociological Review, 41(febrero):16-34.

KLAMER, Arjo (1983), Conversations with Economists. New Classical Economists and Their Opponents Speak Out on the Current Controversy in Macroeconomics. Rowman \& Littlefield Publishers, Savage, MD.

KLAMER , Arjo (1987), 'As If Economists and Their Subjects Were Rational', en John S. Nelson, Alan Megill y Donald McCloskey (eds.) (1987), pp. 163-183.

KLAMER, Arjo; D. N. McCLOSKEY y Robert M. SOLOW (eds.) (1988), The Consequences of Economic Rhetoric. Cambridge University Press, Cambridge.

KUHN, Thomas S. ([1962] 1969), La estructura de las revoluciones cientificas. F.C.E., Madrid, 1982. 
LAMO DE ESPINOSA, Emilio; José María GONZALEZ GARCIA y Cristobal TORRES ALBERO (1994), La sociologia del conocimiento y de la ciencia. Alianza Editorial, Madrid.

LATOUR, Bruno y P. FABRI (1977), 'La Rhétorique de la Science', Actes de la Recherche en Sciences Sociales, 13, 81-95.

MANNHEIM, Karl (1936), Ideología y utopía. Introducción a la sociología del conocimiento. F.C.E., Mexico, 1987.

McCLOSKEY, Donald N. (1985), The Rhetoric of Economics. University of Wisconsin Press, Madison. (Existe versión castellana a cargo de Angeles Conde, publicada por la editorial Alianza, 1990).

MORTARA GARAVELLI, Bice (1988), Manual de retórica. Cátedra, Madrid, 1991.

MUNZ, Peter (1990), 'The Rhetoric of Rhetoric,' Journal of the History of Ideas, 51(1):121-142.

MYERS, Greg (1990a), Writing Biology: Texts in the Social Construction of Scientific Knowledge. University of Wisconsin Press, Madison.

MYERS, Greg (1990b), 'Sociology of Science Without the Sociology', Social Studies of Science, 20:559-563.

MYERS, Greg (1992), 'Textbooks and the Sociology of Scientific Knowledge', English for Specific Purposes, 11(1):3-17.

NELSON, John. S.; Alan MEGILL y Donald McCLOSKEY (eds.) (1987), The Rhetoric of the Human Sciences. University of Wisconsin Press, Madison.

OVERINGTON, Michael A. (1977), 'The Scientific Community as Audience: Toward a Rhetorical Analysis of Science', Philosophy and Rhetoric, 10(2):143-164.

PERELMAN, Chaïm (1990), 'Rhetoric', Encyclopadia Britannica vol. 26, Chicago University Press, Chicago, pg.. 803-810 (15_ed.).

PERELMAN, Chaïm y Lucie OLBRECHTS-TYTECA (1958), Tratado de la argumentación. Gredos, Madrid, 1989.

PINCH, Trevor (1990), 'The Sociology of the Scientific Community', en R. C. Olby, G. N. Cantor, J. R. R Christie y J. S. Hodge (eds.) (1990), Companion to the History of Modern Science. Routledge, Londres, pg. 87-99.

PRELLI, Lawrence J. (1989), A Rhetoric of Science. Inventing Scientific Discourse. University of South Carolina Press, Columbia, SC.

RICHARDS, I. A. (1936), The Philosophy of Rhetoric. Oxford University Press, Londres. ROUSE, Joseph (1993), 'What Are Cultural Studies of Scientific Knowledge?', Configurations, 1(1):1-22.

SIMONS, Herbert W. (ed.). (1989), Rhetoric in the Human Sciences. Sage, Londres. 
SIMONS, Herbert W. (ed.) (1990), The Rhetorical Turn. Invention and Persuasion in the Conduct of Inquiry. The University of Chicago Press, Chicago.

SCHUSTER, John A. y Richard R. Yeo (eds.) (1986), The Politics and Rhetoric of Scientific Method. Reidel, Dordrecht.

TOULMIN, Stephen E. (1958), The Uses of Argument. Cambridge University Press, Londres.

WEIMER, Walter B. (1977), 'Science as a Rhetorical Transaction: Toward a Nonjustificational Conception of Rhetoric', Philosophy and Rhetoric, 10(1):1-29. 